\title{
RETROALIMENTACIÓN FORMATIVA PARA ESTUDIANTES DE EDUCACIÓN A DISTANCIA
}

\author{
(TRAINING FEEDBACK FOR ONLINE STUDENTS)
}

\author{
Fernando Gustavo Lozano Martínez \\ Laura Adriana Tamez Vargas \\ Universidad TEC Virtual, México
}

\section{RESUMEN}

Estudio cualitativo que investiga de qué manera un enfoque de evaluación formativa configura nuevas maneras de retroalimentar a estudiantes de cursos en línea. Participaron 32 profesores de una institución privada que ofrece programas a distancia por Internet. Se diseñó un curso de capacitación para enseñar un modelo de retroalimentación que promueve la evaluación formativa. Los resultados muestran que se puede mejorar la retroalimentación que proporcionan los profesores proveyendo de información al estudiante que tiene que ver con contestar al estudiante las siguientes preguntas a la hora de retroalimentar: ¿Hacia dónde voy? ¿Cuál es el objetivo o la meta? ¿Cómo lo estoy haciendo? ¿Qué sigue después? ¿Cómo se conecta esta actividad con la siguiente? El sentir de los participantes fue positivo, terminaron motivados para incorporar los elementos del modelo a la retroalimentación que dan a sus alumnos.

Palabras clave: retroalimentación, retroalimentación formativa, profesor tutor, cursos en línea.

\begin{abstract}
This qualitative study focuses on researching the following questions: How can a training assessment approach create new ways of providing feedback to students for online courses? 32 teachers from a private institution that offers on line programs via the Internet participated in this study. A training course was designed to teach a feedback model that promoted training assessment. The results show that one can improve teacher feedback by providing the following information to the student, which is based on answering following questions: What am I trying to achieve in my studies? What is the objective or the goal? How am I trying to achieve this goal? How do I connect this activity with the following? The overall reaction of the participants was positive. They demonstrated a certain level of motivation when incorporating the elements of the student feedback model.
\end{abstract}


Keywords: Feedback, training feedback, Academic Tutor, Online courses.

\section{PLANTEAMIENTO DEL PROBLEMA}

La educación a distancia, particularmente en línea, está teniendo mayor aceptación cada día ya que ha representado un recurso que se ajusta a las necesidades de personas que por diferentes motivos no pueden asistir a un modelo presencial. En la educación en línea se sugiere que el estudiante tenga habilidades de autoestudio, autocontrol, autorregulación y que tenga el gusto y la motivación por el estudio continuo, y lo suficientemente motivado para que el estudio sea un interés propio, nada impuesto. Por cada actividad o tarea que el estudiante realice se necesita que su desempeño y/o producción académica sea evaluada y retroalimentada por un profesor tutor quien será su guía en el proceso de aprendizaje. Precisamente en el rubro de la retroalimentación es donde surge la necesidad para realizar el estudio que se encuentran áreas de oportunidad en profesores que dominan su área disciplinar pero muestran carencias en su forma de retroalimentar.

Esta pregunta deben hacerse los profesores ¿Qué tan efectiva es la retroalimentación que doy a los estudiantes? Si únicamente se otorga una calificación numérica o solo se mencionan los errores cometidos, no se enriquecerá el aprendizaje del estudiante.

Holmes y Papageorgiou (2009) realizaron un estudio exploratorio cualitativo para conocer las expectativas, percepciones y usos de la retroalimentación en estudiantes de educación superior de administración turística en el Reino Unido. A los estudiantes se les preguntó qué entendían por retroalimentación, a lo que respondieron que la consideran como mensajes escritos relacionados a la evaluación de una tarea. Los estudiantes comentan que sienten no tener una retroalimentación formativa; les gustaría una retroalimentación que les ayude a mejorar su aprendizaje y sus calificaciones.

En un estudio realizado por Lipnevich \& Smith (2009) enfocado a conocer las perspectivas de los estudiantes sobre la efectividad de la retroalimentación participaron 49 estudiantes en grupos focales. Los autores reportan que los estudiantes comentan que la información acerca de los errores que cometieron y las sugerencias de cómo corregirlos son necesarias para mejorar el aprendizaje, aprecian los comentarios detallados como los más importantes y útiles para su proceso de aprendizaje.

Wanda Bonnel y Heidi Boehm (2011) llevaron a cabo un estudio llamado "Mejorando la retroalimentación para alumnos en línea: tips de enseñanza de 
profesores experimentados". Utilizaron el método exploratorio descriptivo a través de cuestionarios a profesores acerca de su enfoque hacia la retroalimentación. Los resultados muestran que los profesores utilizan rúbricas, criterios y respuestas automatizadas. Mencionan que ser proactivos minimiza los problemas, esto implica guiar en forma oportuna a los estudiantes y aclarar las dudas que se tengan con respecto a la actividad que están realizando. En cuanto al tiempo de retroalimentar, proponen darle una importancia en la agenda; se tiene que planear la retroalimentación de acuerdo al número de estudiantes a los que se tienen que atender. Algunos profesores optan por retroalimentar borradores de las tareas con miras a obtener reportes mejor elaborados.

Precisamente sobre el proceso de retroalimentación que proporciona el profesor tutor al estudiante es que surgió la idea del presente estudio, por una necesidad que semestre a semestre emerge de las encuestas de opinión que se aplican a los estudiantes: mejorar la calidad de la retroalimentación que proporcionan los profesores tutores.

La retroalimentación es un elemento significativo en el proceso de aprendizaje, significa el poder apoyar al estudiante a lograr la meta propuesta de un curso. Sin embargo es un tema del cual los estudiantes no siempre están conformes. Debido a esto surge la pregunta de investigación: ¿De qué manera un enfoque de evaluación formativa configura nuevas maneras de retroalimentar a estudiantes de cursos en línea?

\section{Objetivos del estudio}

Objetivo general: Analizar los foros de discusión del curso en línea "retroalimentación para construcción del conocimiento" ofrecido a 32 profesores de una institución privada que ofrece cursos en línea, con el fin de conocer qué tanto los profesores cambian su estilo de retroalimentación.

Objetivos específicos:

- Analizar en qué forma los participantes llevan a cabo sus retroalimentaciones antes de conocer el nuevo modelo propuesto por los autores Hattie y Timperley (2007).

- Conocer el sentir de los participantes ante el nuevo modelo para saber si consideran que han aprendido algo diferente a la forma de retroalimentación que habían hecho anteriormente, si consideran que el modelo les aporta 
algún valor agregado y si es oportuno implementar el modelo en su práctica docente.

El estudio se realiza en una reconocida universidad que ofrece cursos a distancia por Internet, ante la petición de sus directivos de mejorar el proceso de retroalimentación a los estudiantes. Esto debido a que los mismos alumnos, en encuestas de opinión sobre el desempeño de sus profesores, comentan la necesidad de mejorar la calidad de la retroalimentación.

\section{MARCO TEÓRICO CONCEPTUAL}

Retroalimentar es una actividad clave en el proceso de enseñanza-aprendizaje del alumno, ya que implica darle información que le ayude a cumplir con los objetivos de aprendizaje. No es suficiente con decirle al alumno que su tarea está bien o mal, o corregirle aspectos de formato. La idea es ayudarle a enriquecer su aprendizaje.

Como puede observase en la figura 1, al momento de retroalimentar tenemos que ver la diferencia entre lo que fue solicitado al estudiante en las instrucciones, con lo que entregó, pero no sólo debemos señalar esta discrepancia sino que se requiere que expresemos en forma concreta las áreas de oportunidad que tiene el alumno para aprovechar mejor el aprendizaje (Hattie y Timperley, 2007).

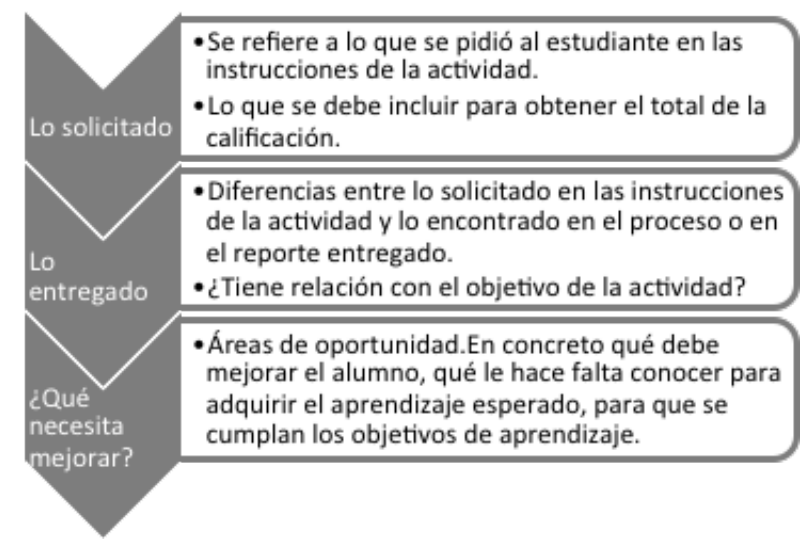

Figura 1. Proceso para decidir qué mensaje se debe escribir al alumno al retroalimentar

\section{¿Qué es retroalimentar?}

La retroalimentación es la información que provee un agente como podría ser: un profesor, un compañero de equipo, un libro, uno mismo, sobre el desempeño 
académico de una actividad de aprendizaje (Hattie y Timperley, 2007). Un profesor posee información profunda sobre el contenido del curso donde es tutor, o por lo menos eso es lo esperado, un compañero de equipo puede proveer una estrategia alternativa para desarrollar una tarea, un libro puede proveer información para clarificar ideas, un consejero puede asesorar al estudiante; así, el aprendiz puede revisar la retroalimentación y considerar corregir las áreas de oportunidad para mejorar el aprendizaje. Retroalimentar, entonces, es la consecuencia que surge después de la presentación de una actividad de aprendizaje.

De acuerdo con Hattie y Timperley (2007) la retroalimentación busca que el alumno se dé cuenta de la discrepancia que hay entre lo que comprendió y lo que debió haber comprendido, o cómo se ha desempeñado para cumplir con el objetivo de aprendizaje de cada actividad. Dicho de otra manera, que vea la diferencia entre la actividad que entregó y la que debió haber sido entregada, o rectificar el proceso que llevó a cabo con el que debió haber realizado para cumplir con el objetivo o competencia de aprendizaje.

Retroalimentar una actividad de aprendizaje es la acción en que el profesor proporciona al estudiante información enfocada a mejorar un producto o proceso académico, mientras que cuando se otorga solo una calificación se denomina evaluación sumativa, y no mejora el aprendizaje del estudiante. El tipo de retroalimentación enfocada a proveer información sobre el proceso llevado a cabo por el estudiante en la realización de una tarea, ya sea para aclarar un conocimiento o para adquirir una habilidad, se le denomina formativa. Shute (2008, citado por Jonsson, 2012) describe la retroalimentación formativa como la información comunicada por el profesor al estudiante que intenta modificar su pensamiento o su comportamiento para mejorar el aprendizaje. No es suficiente con proporcionar una calificación numérica, el estudiante espera información que le aporte algo que le ayude a comprender un conocimiento nuevo, que le lleve a saber algo más.

Para que la retroalimentación sea efectiva, Shute (2008, citado por Jonsson, 2012) menciona que la información preferentemente debería estar enfocada a la tarea elaborada por el estudiante y no relacionada con características personales del estudiante. Jonsson comenta que si los estudiantes no utilizan la retroalimentación que reciben de sus profesores es porque en ocasiones la encuentran poco útil, los estudiantes no están conformes con la retroalimentación que reciben: Sadler (1989, citado por Jonsson, 2012) encuentra tres premisas que los estudiantes necesitan tener en las retroalimentaciones: (1) Deben saber el objetivo de la actividad, (2) recibir retroalimentación con referencia a algún estándar, (3) conocer algunas estrategias para modificar la actividad de manera que se cumpla con las expectativas. 
Otro aspecto importante es el tiempo en que se entrega la retroalimentación ya que esta debe ser oportuna, proporcionarla de tal manera que pueda ser utilizada por el estudiante en la mejora de la realización de las actividades posteriores del curso o modulo y no enviarla cuando ya haya terminado el mismo (Jonsson, 2012).

La función más importante de la retroalimentación de acuerdo a Black y William (1998, citado por Shute, 2008) es la dirección y facilitación. Retroalimentación directiva es aquella que informa al estudiante lo que necesita ser ajustado o revisado, esta retroalimentación tiende a ser más específica, provee comentarios y sugerencias que guían al estudiante en su propia revisión y conceptualización.

Evans (2013) encontró que la retroalimentación se ve como una herramienta correctiva, sin embargo, esta debería ser vista como una herramienta que ayude al estudiante para que clarifique sus dudas y como un medio para mejorar su aprendizaje.

De acuerdo al modelo de retroalimentación (ver figura 2) de Hattie \& Timperley (2007), la retroalimentación efectiva responde a tres preguntas que deben estar muy claras para el alumno:

La primera pregunta que debe estar clara para el alumno es: ¿Cómo voy? ¿Hacia a dónde estoy yendo? Esto quiere decir que en la retroalimentación debemos informar al alumno si está cubriendo los objetivos de aprendizaje del curso y de cada actividad en particular. Esto sitúa al alumno en los objetivos del curso, ya que de lo contrario está elaborando las actividades sin un rumbo definido.

La segunda pregunta que debe estar clara para el alumno es: ¿Cómo lo estoy haciendo? Esto significa informar al estudiante tanto el desempeño que está teniendo en sus actividades de aprendizaje, como en el resultado de los productos entregados. El cómo lo estoy haciendo tiene que ver con la forma en que el estudiante va construyendo las actividades de aprendizaje, es decir proveer información en el proceso de construcción, de tal manera que el alumno pueda hacer ajustes pertinentes a las acciones que hace para lograr con una meta determinada.

La tercera pregunta que debe estar clara para el alumno es: ¿Qué sigue después de esto? Es decir, nosotros como profesores debemos informar al alumno en nuestra retroalimentación cómo va a conectar la actividad que se está evaluando con la siguiente, no son productos aislados, cada actividad debe tener un objetivo claro encaminado a cumplir los objetivos del curso (Hattie y Timperley, 2007). 


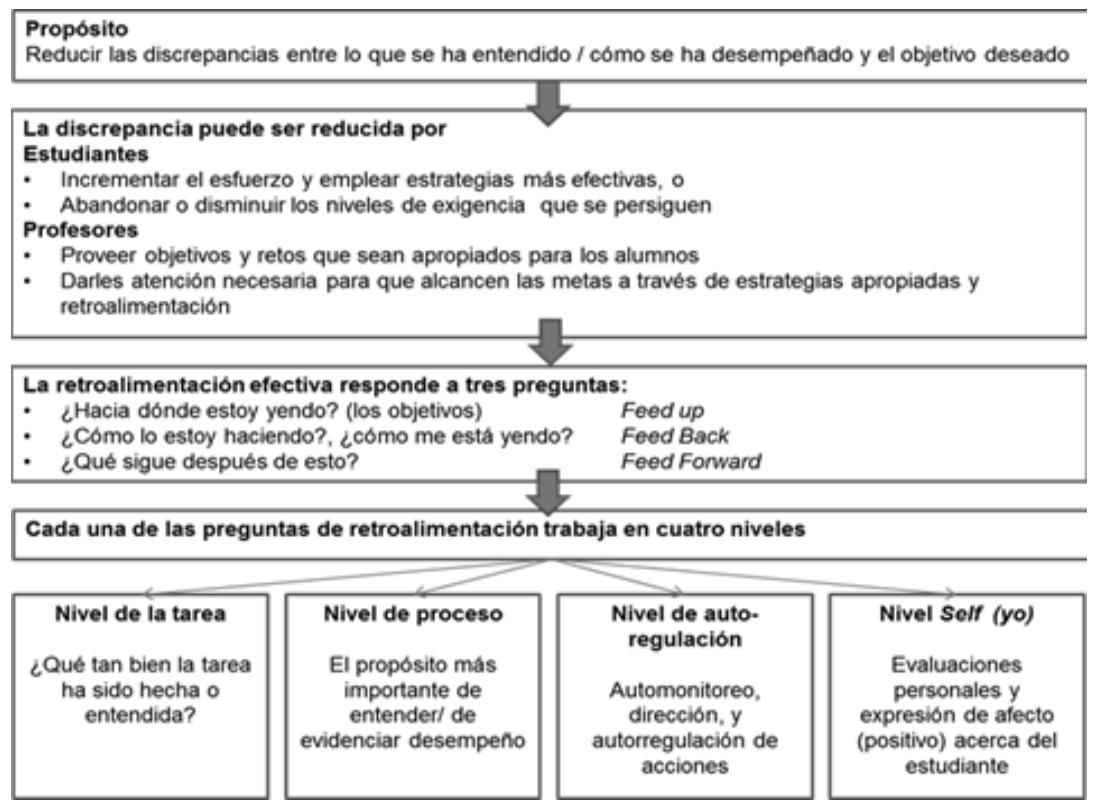

Figura 2. Modelo de retroalimentación de Hattie y Timperley (2007). Traducido al español por la Dra. Katherina Gallardo

¿A qué niveles se puede enfocar la retroalimentación? De acuerdo al modelo de retroalimentación de HattieyTimperley(2007), se puedeenfocar la retroalimentación a uno de los siguientes niveles:

- Al nivel de la tarea, que se enfoca en ver qué tanto se comprendió lo que se tenía que hacer y qué tan bien se hizo el producto que se solicitó.

- Al nivel del proceso, que es donde se evidencia cómo fue el desempeño.

- Al nivel de autorregulación, tiene que ver con el automonitoreo, autodirección, y autorregulación de acciones.

- Al nivel del yo, de las emociones, es donde se hacen evaluaciones personales y se escriben expresiones motivacionales a los estudiantes.

De acuerdo a Hattie \& Timperley (2007), ejemplos de retroalimentación enfocados al nivel de la tarea son: "Necesitas incluir más acerca del tratado de Versalles"; "Hace falta profundizar en las características de los recursos educativos abiertos"; "En esta sección es preciso describir los niveles de apropiación tecnológica”. 
Ejemplos de retroalimentación enfocados al nivel del proceso, utilizado para crear un producto o completar una tarea son: "Es recomendable editar la primera parte del marco teórico con el fin de que se puedan entrelazar las ideas de los tres autores que se manejan en esta sección. Podrías hacer uso de un organizador o mapa conceptual para que se te haga más fácil unir las ideas de los tres autores". "La metodología quedaría más entendible para el lector si se considera describir el procedimiento utilizado tal como lo vimos en el ejemplo descrito en el video explicativo".

Ejemplos de retroalimentación enfocados al nivel de autoregulación son: "Para este momento ya conoces el formato de encabezado que debe llevar cada instrumento de recolección de datos. Verifica si has incluido todos los datos solicitados en el instrumento que has elaborado"; “¿Consideras que las preguntas incluidas en el instrumento servirán para responder a tu pregunta de investigación?”.

Ejemplos de retroalimentación enfocados al nivel del yo son: "Muy bien, eso es correcto"; "Eres buen estudiante".

\section{¿Qué implica una retroalimentación asertiva?}

Una retroalimentación asertiva es aquella que le aporte al estudiante información específica, detallada, individualizada, en tiempo, enfocada a un nuevo aprendizaje, detectando áreas de oportunidad en las que puede mejorar; además una buena retroalimentación reduce la distancia entre lo que comprendió el estudiante y lo que debió haber comprendido.

\section{METODOLOGÍA}

\section{El enfoque de la investigación}

El estudio se llevó a cabo bajo un enfoque cualitativo. La investigación cualitativa se enfoca a la recolección, análisis e interpretación de datos, no numéricos, visuales y narrativos con el fin de obtener reflexiones de un fenómeno en particular. La investigación cualitativa examina un fenómeno en particular sin una guía acerca de lo que pudiera ser o pudiera no ser verdad acerca de ese fenómeno o su contexto. En investigación cualitativa el contexto no es controlado o manipulado por el investigador. El número de participantes tiende a ser pequeño porque son muestras recolectadas con base en un propósito (Taylor y Bogdan, 1987). En esta investigación los datos se tomaron de los foros de discusión del curso de capacitación 
"retroalimentación para la construcción del conocimiento", diseñado para persuadir a los profesores a cambiar su forma de retroalimentar a sus estudiantes.

El procedimiento llevado a cabo en el estudio es el propuesto por Gay, Mills, y Airasian (2006, p. 401):

- "Identificar un tópico de investigación. El investigador identifica un tópico o estudio de interés para investigar. El tema debe estar delimitado para que sea más viable.

- Revisar literatura. El investigador examina investigación ya existente para identificar información útil y estrategias para llevar a cabo el estudio.

- Seleccionar participantes. El investigador debe seleccionar a los participantes que serán las fuentes de información. Los participantes son seleccionados (no al azar) y son usualmente pocos en comparación con la investigación cuantitativa.

- Recolectar datos. El investigador recolecta datos de los participantes. Datos cualitativos se obtienen a través de entrevistas, observaciones y artefactos.

- Analizar e interpretar datos. El investigador analiza los temas y resultados de los datos recolectados y provee interpretaciones de los datos.

- Reportar y evaluar la investigación. El investigador integra los datos cualitativos en forma narrativa y visual".

\section{Muestra}

El número de participantes tiende a ser pequeño porque son muestras recolectadas con base en un propósito (Gay, Mills y Airasian, 2006).

Participaron 32 profesores en línea, de educación superior, divididos en dos grupos tutoriales, cada uno con un facilitador. Los profesores pertenecen a una institución privada que ofrece cursos presenciales y a distancia a través de Internet. Participaron profesores de la Escuela de Graduados en Educación, de la Dirección de Graduados en Administración, del Programa de Graduados en Ingenierías y Tecnologías, del Programa de Apoyo a los Campus del Sistema y diseñadores instruccionales del área de Tecnología Educativa. 


\section{Validez}

Para dar validez a los resultados se utilizó la triangulación que es una estrategia para verificar la consistencia entre los datos recabados. Triangular significa dar un apoyo a un resultado, mostrando que un determinado resultado, obtenido por ejemplo en una entrevista, de una observación o de un documento, coincide con otro resultado obtenido en otra observación, o en otra entrevista con otra persona. La triangulación de datos se refiere simplemente a utilizar diferentes fuentes de datos, para ver las convergencias o divergencias (Mathison, 1988). En el estudio la triangulación se llevó a cabo entre profesores de cada área, entre profesores con diseñadores instruccionales, y entre los resultados y la teoría.

Los resultados de este estudio sirvieron a los directivos de la institución para ofrecer el mismo curso, a través del departamento de capacitación de la institución, hacia todas las áreas de la misma universidad con el fin de mejorar el tipo de retroalimentación que reciben los estudiantes.

Para el estudio se diseñó, desarrolló e implementó un curso de capacitación en línea llamado "retroalimentación para la construcción del conocimiento", con el fin de conocer el impacto que pudiera llegar a tener ante un grupo de profesores el conocer un modelo de retroalimentación que pudieran aplican en su práctica docente.

En el curso inicialmente se sensibilizó a los participantes acerca de ¿Qué se necesita saber para retroalimentar un curso? Para ello los participantes analizaron los objetivos generales de un curso diferente al curso donde son profesores, también analizaron los objetivos particulares, los módulos en los que está dividido, los contenidos que constituyen cada módulo, las actividades de cada módulo y los criterios o rúbricas de evaluación. Además, revisaron ejemplos de retroalimentación para identificar los elementos del modelo de Hattie y Timperley (2007). Finalmente reelaboraron retroalimentaciones que ellos mismos habían proporcionado en períodos anteriores pero ahora ajustándolos al modelo propuesto. Se abrió un foro para reflexiones finales. Las unidades de análisis fueron los foros de discusión donde se desarrollaron las actividades de aprendizaje.

\section{Participantes}

El grupo se formó de 32 profesores los cuales fueron seleccionados de la siguiente manera: se informó sobre el curso de capacitación a los directores de cada área quienes invitaron a algunos profesores a participar en el curso. El criterio de 
selección fue la experiencia de más de dos años como profesores tutores en línea y sus altos estándares de desempeño.

De acuerdo a la información que se provee en el portal de esta institución, se ofrece educación de calidad utilizando modelos educativos innovadores, redes de aprendizaje y tecnologías de información avanzadas, para contribuir a la integración y desarrollo de las comunidades de habla hispana. También se diseñan cursos que propician el desarrollo de competencias y la gestión de conocimientos, utilizando para ello la tecnología de Internet. Ofrece programas de posgrado, educación continua y desarrollo social para más de 80.000 alumnos en todo el continente americano, así como para empresas y organizaciones que requieren cubrir necesidades de capacitación.

Los cursos son diseñados por profesores expertos en el área de conocimiento y por un equipo interdisciplinario de especialistas en el uso de recursos didácticos y tecnológicos para la educación a distancia. Los contenidos se presentan a través de herramientas tecnológicas como videos, animaciones, gráficos, simulaciones y recursos didácticos tales como materiales digitales, lecturas de apoyo, ligas de interés, glosarios, analogías que permiten aprovechar las características del Internet y asegurar el aprendizaje activo y significativo del alumno.

El estudiante tiene una participación activa en el proceso de aprendizaje, realizando actividades de autoestudio y colaborativas que le permiten un aprendizaje significativo para su desempeño profesional. En un curso basado en Internet el alumno no se encuentra solo; está acompañado durante todo el curso por un profesor que facilita su aprendizaje y el de sus compañeros a través de una asesoría personalizada y su participación orientadora en las actividades de autoestudio y colaborativas.

\section{DISEÑO DEL CURSO DE CAPACITACIÓN}

El curso de capacitación en línea se llamó Retroalimentación en la Construcción del Conocimiento, con una duración propuesta de tres semanas. La introducción al curso se enfoca en la importancia de provocar que el aprendizaje del alumno sea lo más importante para un profesor. Hattie y Timperley (2007) en su artículo "El Poder de la Retroalimentación" dicen que la primera pregunta que se debe hacer el docente al escribir una retroalimentación es: ¿Qué tan efectiva es la retroalimentación que proporcionamos a los estudiantes? 
Insertos en un sistema de educación virtual donde prácticamente todas las interacciones comunicativas entre profesores y alumnos ocurren por algún medio escrito, la redacción de cada mensaje se vuelve nuestra tarjeta de presentación. De ahí la importancia del fondo de las comunicaciones, pero también de la forma en que se hacen. ¿Qué tanto se ocupa en la revisión de la forma en que lo decimos?

Posiblemente somos de la idea de que no hay mucho que aprender de cómo decir las cosas, pero si tomamos en cuenta que el receptor de nuestros mensajes no está viendo los gestos de la cara, la postura del cuerpo, ni escuchando el tono de la voz, etc, nuestra idea puede cambiar. Cuando utilizamos la escritura como herramienta para comunicarnos cada palabra puede tener un significado diferente para nuestro receptor.

En las encuestas que contestan los alumnos al terminar cada curso, es fácil encontrar el comentario de que algunos profesores retroalimentan de forma cortante, fría y distante. Y lo que sucede es que un mismo mensaje se puede decir de diferentes maneras, no es lo mismo decir: "Estás mal, no entendiste lo que tenías que hacer, no leíste bien las instrucciones", a decir: "Considero que el reporte presentado puede ser mejorado, sobretodo en el apartado correspondiente a los objetivos específicos en donde hace falta mencionar dónde realizarán la acción que proponen. Sugiero revisar los ejemplos que adjunto".

Las actividades de aprendizaje de este curso tienen su entrega en foros de discusión de la plataforma. El participante tiene que elaborar todas las actividades de aprendizaje para acreditar el curso.

Los objetivos de aprendizaje fueron:

- Conocer los elementos básicos que contienen los cursos de esta institución.

- Conocer el modelo de capacitación de Hattie y Timperley.

- Analizar retroalimentaciones realizadas en un curso para encontrar fortalezas y debilidades, utilizando el modelo de retroalimentación de Hattie y Timperley.

- Identificar los elementos del modelo de retroalimentación de Hattie y Timperley en el curso que se trabaja como tutor, con el fin de realizar una propuesta, incluyendo los cambios que se puede innovar a la forma de retroalimentar. 
- Enlistar las acciones que se espera de un profesor de nuestra institución realice, enfocadas a la retroalimentación a los alumnos.

\section{Método de aprender en el curso de capacitación}

Para aprender una nueva forma de proporcionar retroalimentación a los estudiantes, el participante de este curso necesita conocer el qué debe hacer, el cómo debe hacerlo y cuál es la intención de ello.

¿Qué necesito saber? Se necesita conocer el modelo de retroalimentación propuesto por los autores Hattie y Timperley, conocer el curso al que pertenecen las actividades que se van a retroalimentar, conocer el o los objetivos del curso, los módulos en que se dividen los contenidos, las actividades de aprendizaje, la metodología y la forma de evaluar.

¿Cómo lo voy a lograr? Una vez que el participante conoce el modelo de retroalimentación deHattieyTimperleyylas características delcursoalquepertenecen las actividades de aprendizaje que se retroalimentaron, va a cotejar el modelo de retroalimentación con las evaluaciones que hizo un tutor a diferentes actividades de aprendizaje con el fin de ver cómo se podría mejorar la retroalimentación. Finalmente, el participante elabora un plan para mejorar la retroalimentación que proporciona a sus propios alumnos en el curso que le corresponde tutorear.

\begin{tabular}{|c|c|c|c|}
\hline Tiempo & Temas a estudiar/actividades & Material a utilizar & Producto \\
\hline \multirow{2}{*}{ 01:00 } & $\begin{array}{l}\text { Revisar la información del curso y } \\
\text { presentarte en el foro. }\end{array}$ & Página del curso & \multirow[t]{2}{*}{ No aplica. } \\
\hline & Ver video introductorio. & Video & \\
\hline \multirow[t]{2}{*}{ 06:00 } & Modelo de retroalimentación. & $\begin{array}{l}\text { Artículo de Hattie y } \\
\text { Timperley localizado } \\
\text { en recursos de apoyo. } \\
\end{array}$ & No aplica. \\
\hline & $\begin{array}{l}\text { Cotejar elementos de un curso } \\
\text { de la universidad con elementos } \\
\text { del modelo de retroalimentación } \\
\text { propuesto. }\end{array}$ & \begin{tabular}{|l} 
PDF con elementos \\
de un curso de la \\
universidad en \\
recursos de apoyo.
\end{tabular} & $\begin{array}{l}\text { Análisis de } \\
\text { cotejo en el foro } \\
\text { correspondiente. }\end{array}$ \\
\hline 04:00 & $\begin{array}{l}\text { Revisar y analizar cinco ejemplos } \\
\text { de retroalimentación del curso } \\
\text { Tecnología e Innovación en } \\
\text { Educación con el modelo de Hattie y } \\
\text { Timperley. }\end{array}$ & $\begin{array}{l}\text { Ejemplos en recursos } \\
\text { de apoyo. }\end{array}$ & $\begin{array}{l}\text { Reporte en } \\
\text { Word. Subir } \\
\text { archivo en el foro } \\
\text { correspondiente. }\end{array}$ \\
\hline
\end{tabular}




\begin{tabular}{|c|c|c|c|}
\hline Tiempo & Temas a estudiar/actividades & Material a utilizar & Producto \\
\hline \multirow[t]{2}{*}{ 05:00 } & $\begin{array}{l}\text { Enlistar datos del curso donde } \\
\text { tutoreas. }\end{array}$ & $\begin{array}{l}\text { Datos de tu curso. } \\
\text { Nota: En caso de } \\
\text { que aún no tengas } \\
\text { un curso donde } \\
\text { seas profesor } \\
\text { tutor(a) puedes } \\
\text { utilizar los datos del } \\
\text { curso Tecnología } \\
\text { e Innovación en } \\
\text { Educación que se } \\
\text { encuentra localizado } \\
\text { en recursos de apoyo. }\end{array}$ & \multirow[t]{2}{*}{$\begin{array}{l}\text { Reporte en Word } \\
\text { con datos de } \\
\text { curso y modelo } \\
\text { innovador. Subir } \\
\text { archivo en foro } \\
\text { correspondiente. }\end{array}$} \\
\hline & $\begin{array}{l}\text { Elaborar un modelo o estrategias de } \\
\text { retroalimentación para tu curso. }\end{array}$ & $\begin{array}{l}\text { Se realiza con base en } \\
\text { lo visto en este curso } \\
y \text { tu experiencia } \\
\text { personal. }\end{array}$ & \\
\hline 03:00 & $\begin{array}{l}\text { Deberes para cumplir la labor } \\
\text { docente. }\end{array}$ & $\begin{array}{l}\text { Artículo de Siruana } \\
\text { localizado en } \\
\text { recursos de apoyo. }\end{array}$ & $\begin{array}{l}\text { Foro } \\
\text { correspondiente. }\end{array}$ \\
\hline 01:00 & Reflexiones finales. & $\begin{array}{l}\text { La experiencia del } \\
\text { curso. }\end{array}$ & $\begin{array}{l}\text { Foro } \\
\text { correspondiente. }\end{array}$ \\
\hline
\end{tabular}

Tabla 1. Organizador del curso de capacitación

Las actividades de este curso no tienen una ponderación. Para aprobar el curso se requiere que el participante elabore las cinco actividades de aprendizaje.

Actividad I: Lo que necesito saber para retroalimentar.

- Leer el artículo: The Power of Feed Back.

- Revisar los elementos de un curso de la universidad que se deben conocer para poder proporcionar una retroalimentación efectiva, e inferir en cuáles de ellos se pueden aplicar al modelo de retroalimentación de Hattie y Timperley. Este análisis lo subes al foro correspondiente. 
Actividad II: Revisar ejemplos de retroalimentación.

- Revisa los cinco ejemplos de retroalimentación localizados en Recursos de Apoyo. Revisa cada ejemplo, contesta cada una de las preguntas siguientes dentro de un archivo de Word, y súbelo en el foro correspondiente:

- De acuerdo a tu opinión ¿para qué crees que se elaboró esta actividad de aprendizaje?

- ¿Qué esperarías encontrar en la actividad de aprendizaje para que se cumpla el objetivo general y particular del curso?

- ¿Es una retroalimentación o no?

- ¿En qué basas tu respuesta anterior?

- ¿Cuáles son las fortalezas y debilidades que identificas?

- ¿Crees que la retroalimentación fue enfocada al objetivo general del curso?

- ¿Crees que la retroalimentación fue enfocada al objetivo particular del curso?

- ¿Es útil la retroalimentación para la siguiente actividad del curso?

- ¿Puedes inferir si la retroalimentación fue generada a partir de criterios de evaluación?

- ¿La retroalimentación tiene alguna característica emotiva? En caso afirmativo ¿̇podrías decir cuál fue? ¿Qué es lo que dice exactamente?

- ¿QQué le agregarías tú a esta retroalimentación?

- ¿Crees que la actividad de aprendizaje que se evalúa amerita ser retroalimentada bajo el modelo de Hattie y Timperley?

- Retroalimenta a uno de tus compañeros siguiendo el modelo de Hattie y Timperley. Retroalimenta a alguien que no haya sido retroalimentado. 
Actividad III: Planeando mi estilo de retroalimentación.

- En un archivo de Word menciona los siguientes datos y súbelo al foro correspondiente:

- Nombre del curso donde eres profesor tutor.

- ¿Cuál es el objetivo general del curso?

- ¿Cuáles son los objetivos particulares?

- ¿EEn cuántos módulos está dividido el curso?

- Enlista el nombre de los contenidos de cada módulo.

- Elabora una síntesis de cada uno de los contenidos.

- Describe cada una de las actividades de aprendizaje.

- ¿QQué objetivo crees que se cumple con cada actividad?

- ¿Tienen criterios de evaluación o rúbrica de evaluación cada una de las actividades? En caso afirmativo ¿cuáles son?

- En el mismo archivo de Word elabora un modelo o describe estrategias de retroalimentación para el curso que tutoreas, con el fin de realizar una propuesta incluyendo los cambios que puedes innovar a tu forma de retroalimentar. Toma en cuenta el tiempo que tienes para retroalimentar y la cantidad de alumnos que atiendes, así como el número de tareas que tienes que revisar, que dependerá si las actividades son individuales o en equipo. Nota: En caso de que aún no tengas un curso donde seas profesor tutor(a) puedes utilizar los datos del curso Tecnología e Innovación en Educación que se encuentra localizado en recursos de apoyo.

- Compártelo en el foro correspondiente.

- Retroalimenta a uno de tus compañeros, utilizando el modelo de Hattie y Timperley. Retroalimenta a alguien que no haya sido retroalimentado. 
Actividad IV: Deberes de la práctica docente.

- Lee el material:

- Siruana, J.C. Ética de las profesiones. Material inédito proporcionado en el curso presencial Ética de las Profesiones ofrecido del 26 al 31 de julio de 2004 en el ITESM - Campus Monterrey, por el Dr. Juan Carlos Siruana, profesor de las áreas de Filosofía Moral y Filosofía Política en la Universidad de Valencia, España. Material utilizado con permiso del autor.

- Enlista de 5 a 10 deberes que tú consideras es necesario llevar a cabo para cumplir cabalmente con tu labor como docente, así mismo menciona aquello que se te hace difícil de llevar a cabo y por qué.

- Comparte tu lista en el foro correspondiente.

- Retroalimenta a uno de tus compañeros.

Actividad V: Reflexiones finales.

Entrar al foro de cierre y hacer una reflexión personal tomando en cuenta las siguientes preguntas:

- ¿'Tiene un nuevo sentido para ti la retroalimentación?

- ¿ंTe motiva a hacer cambios en tu forma de retroalimentar?

- ¿En qué tipo de actividades consideras que se puede utilizar el modelo de retroalimentación de Hattie y Timperley?

- ¿Qué adaptaciones podrías hacer al modelo de retroalimentación de Hattie y Timperley para que sea un modelo viable cuando se atiende a un grupo numeroso de estudiantes?

- ¿Cuáles son las fortalezas del modelo de retroalimentación de Hattie y Timperley?

- ¿Cuáles son las debilidades del modelo de retroalimentación de Hattie y Timperley?

- ¿Con qué te quedas de este curso? ¿Qué aprendiste? 


\section{RESULTADOS, INTERPRETACIÓN Y ANÁLISIS}

Del análisis de los foros emergen las siguientes categorías: "motivados para el cambio", "adaptaciones del modelo de Hattie y Timperley para cursos masivos", y "el antes y el después".

\section{Motivados para el cambio}

La apertura, inquietud, motivación para llevar a la práctica un método diferente para retroalimentar se percibió desde el momento en que conocieron el modelo de Hattie y Timperley, ya que de una forma clara vieron la utilidad que tendría para los estudiantes el proveer información que apoyara para el cambio de conocimientos, actitudes, habilidades, valores con el fin de mejorar el aprendizaje. En este sentido un participante menciona:

Siempre he buscado hacer retroalimentaciones de calidad, pero ahora que conozco el modelo me doy cuenta que me hacían falta muchos elementos por cubrir. Ya he iniciado el uso de este modelo, y aunque sé que iré mejorando paulatinamente, ya he tenido un impacto favorable en mis alumnos.

El modelo nos sitúa en preguntas clave cuyas respuestas es lo que el estudiante quiere saber acerca de cada actividad de aprendizaje: ¿Hacia dónde voy?, ¿Cómo me desempeñé?, ¿Qué sigue? Hacia dónde voy tiene que ver con los objetivos del curso, es ver en qué medida la actividad que se está evaluando promueve a que se logre el objetivo del curso; cómo me desempeñé implica mencionar detalles que tienen que ver con la evaluación formativa y sumativa; formativa en cuanto se hacen señalamientos precisos de las fortalezas y debilidades que el estudiante llevó a cabo en la construcción de la tarea, y sumativa en donde se le asigna el puntaje obtenido con referencia a un estándar de criterios de evaluación prestablecidos.

Por lo general, en los criterios que se proporcionan en los cursos están enfocados a evaluar la tarea y pocos son los que incluyen el proceso. $\mathrm{Y}$ aunque se recomienda de forma general aportarle algo al alumno sobre la experiencia del profesor no se destacan los niveles de retroalimentación a los que se enfoca el modelo. La retroalimentación puede enfocarse al producto solicitado. Puede incluir direcciones más precisas: "Muy bien elaborado el ensayo, resalto la manera tan personal de conducir al lector sobre las estrategias que utiliza un profesor para preparar sus clases". La retroalimentación puede ser enfocada al nivel de autorregulación, incluyendo habilidades de autoevaluación: "Con base en los criterios de evaluación coteja el reporte y señala tus fortalezas y debilidades". La retroalimentación puede ser enfocada a la persona: "Felicidades, estás aprovechando muy bien la oportunidad 
que tienes de estudiar una maestría", alienta a continuar con el proceso, impacta positivamente ya que los alumnos se esfuerzan más, aunque algunos perciben que se les da "aliento" por ser clasificados como estudiantes de bajo desempeño. Referente a estas ideas uno de los comentarios de los participantes resalta lo siguiente:

...para mí lo más relevante ha sido la importancia de ubicar al alumno con la actividad siguiente para que vea el sentido de su trabajo y la repercusión que tiene. Y claro, también cambió mi perspectiva de la relevancia de lo que se coloca en la retro. Los alumnos están ansiosos por recibir los comentarios de nosotros tutores, para ellos mejorar en sus próximas entregas y subir sus calificaciones. Y si les puedo dar estas 4 partes, siento que se sentirán más satisfechos y contentos con mi trabajo, lo que me motiva a hacerlo. De hecho, ya inicié a utilizar el modelo y ahora me doy cuenta de la importancia de cada actividad y cómo se conectan unas con otras.

Cada tutor inició cambios en la forma de retroalimentar a sus estudiantes, incluyendo los elementos que no tenían consciente como parte integral de la retroalimentación. Ahora incluyen aspectos relacionados con la razón de ser de la actividad, así como aquellos referentes a detalles específicos sobre su desempeño. Con esta nueva forma de retroalimentar se le da un seguimiento y una relación entre los objetivos del curso y las actividades que los alumnos están realizando.

Este modelo permite personalizar la retroalimentación. Cuando se utilizan rúbricas de evaluación, en ocasiones se escriben mensajes de retroalimentación muy similares a los equipos. Al tener ya unos lineamientos se pierde la originalidad y nos acoplamos a lo que hay que revisar. El modelo de Hattie y Timperley nos permite discernir más lo que ocupan nuestros alumnos, al conocerlos mejor. Asimismo, como docentes, nos ayuda a ser más receptivos de las cualidades y áreas de oportunidad de los alumnos, y nos da una guía de cómo ayudarlos, enfocándonos en áreas prioritarias.

Entre las fortalezas del modelo podemos mencionar que abarca las partes importantes que integran el aprendizaje de un alumno: tarea, proceso, regulación y el yo. Haber entregado lo solicitado le dará el primer cumplimiento, mientras que haber llevado a cabo el procedimiento indicado de manera correcta le dará el segundo cumplimento. Para lograrlo en el punto de la autorregulación debe hacerse mención en como rehacer aquellas acciones que le fueron evaluadas como deficientes, de esta forma poder vivenciar una autoevaluación. Para cerrar una retroalimentación completa incluyendo el "yo" se deben incluir mensajes motivacionales, para que al estar lejos, se sienta acompañado, con apoyo. 
La estructura ayuda a visualizar en dónde estamos y hacia dónde nos dirigimos, así el alumno tiene más clara la relación entre cada tema para que al final se lleve un valioso aprendizaje. Además, no nada más se centra en lo académico, también lo emocional es importante, el brindarle al alumno confianza, animándolo a seguir adelante.

\section{Adaptación del modelo de Hattie y Timperley para cursos masivos}

Retroalimentar a un nivel muy detallado implica mucho tiempo para llevarlo a cabo. La preocupación de los profesores es cómo hacer para aplicar el modelo propuesto sobre todo cuando la cantidad de alumnos que se retroalimentan es grande, alrededor de 100 estudiantes, y con un promedio de 10 actividades por curso, alguna de ellas individuales, lo cual lo hace aún más complicado.

El sentir de algunos de los participantes:

Ubicar los niveles en los cuales el alumno tiene un muy buen desempeño, un desempeño medio y un desempeño bajo, y enfatizar la retroalimentación principalmente en esos rubros; en ocasiones es complicado retroalimentar cada uno de los cuatro niveles de las tres preguntas a responder que proponen estos autores. Otra opción es verificar con detalle el objetivo de la actividad y analizar cuál nivel es el que más se espera desarrollar en los alumnos.

Considerar el uso de formatos y plantillas, bien hechos, es decir, considerando las 4 partes de la retroalimentación sugeridas por los autores, pero con cierta flexibilidad para poderla personalizar. Por ejemplo, que los criterios de evaluación no solo incluyan evaluar la tarea y el proceso, también incluyan ya acciones predeterminadas, con la flexibilidad de elegirlas, para dar retroalimentación al nivel de autorregulación y la persona.

Sería muy extenso trabajar cada una de las 3 preguntas del modelo en los 4 niveles porque serían básicamente 12 preguntas a responder, por lo que se pudiera trabajar a manera centrada y concreta las 3 preguntas del modelo a nivel general, o bien, para cada actividad en específico, adentrar en los criterios de evaluación para colocar adentro de estos las tres preguntas del modelo para no hacer más extensa la retroalimentación, sino más eficiente y efectiva.

Cuando se tienen grupos masivos los profesores piensan en estrategias que les ayuden a maximizar el tiempo, entre estas están el elaborar plantillas genéricas en donde se escriban a priori, junto a los criterios de evaluación, las respuestas a las preguntas que proponen Hattie y Timpeley, en concreto las preguntas ¿Hacia dónde voy? y ¿Qué sigue?, para dejar la pregunta ¿Cómo me desempeñé? para ser respondida específicamente a cada alumno en particular, o a cada equipo si la actividad fue grupal. 
El sugerir implementar en forma genérica las respuestas a las preguntas ¿Hacia dónde voy? y ¿Qué sigue? es debido a que para todos los alumnos es la misma información, no cambia. Sin embargo, para la pregunta ¿Cómo me desempeñé? sí se tiene que desarrollar una descripción que le informe al alumno cómo ha sido su desarrollo, tanto en el proceso durante el tiempo en el que se desarrolló la actividad, como en el producto final. Hablando del proceso, es importante especificar lo que hizo en forma correcta, mencionar si se está cumpliendo con el objetivo de aprendizaje, si se están tomando en cuenta las instrucciones, sobre todo si han sido comprendidas en forma correcta, esto para disminuir la discrepancia entre lo que dicen las instrucciones de la actividad y lo que el alumno comprendió.

En este entorno de cursos masivos, llevar a la acción esta retroalimentación con el nivel de detalle mencionado anteriormente en actividades individuales, cuando se atienden a 120 alumnos, se convierte en un reto. No necesariamente se tienen que retroalimentar todas las actividades del curso bajo esta lupa, se pueden seleccionar aquellas que tengan más ponderación o que sean las más significativas del curso.

\section{El antes y el después}

En el mismo tiempo en que se llevó a cabo el curso de capacitación para promover una retroalimentación formativa los participantes tuvieron la posibilidad de actualizar su forma de retroalimentar, haciendo cambios precisos en su estilo de retroalimentar, incluyendo las preguntas propuestas por el modelo de Hatttie y Timperley (2007): ¿̇hacia dónde voy?, ¿Cómo lo estoy haciendo? y ¿Qué sigue? enfatizando alguno de los niveles de retroalimentación: a la tarea, al proceso, a la autorregulación o al yo. Sobre esto, uno de los participantes comenta:

Aprendí a fortalecer las retroalimentaciones incluyendo en ellas información más precisa y detallada que antes no mencionaba y que mejorará sustancialmente los siguientes trabajos académicos presentados por los estudiantes.

Es necesario proporcionar información al estudiante que ayude para hacer cambios en su conocimiento, habilidades, actitudes con el fin de mejorar el aprendizaje es un cambio que se percibe en las retroalimentaciones que elaboran los participantes. Uno de los comentarios de los participantes lo enfatiza de la siguiente manera:

Conocer este modelo me deja una mejor idea de la estructura de la retroalimentación efectiva, de cómo relacionar los objetivos del curso con el desempeño de los estudiantes y como motivarlos a esforzarse más. La retroalimentación es mucho más que una lista de cotejo, es nuestro deber como formadores dar retroalimentaciones integrales que provoquen un deseo continuo de mejora y transformación educativa. 
Algunos consideraban a la retroalimentación como un elemento ajeno al proceso de enseñanza-aprendizaje, concepción que fue cambiando con el desarrollo del curso y que podemos apreciar en el siguiente comentario:

\begin{abstract}
Ahora veo la manera en que la retroalimentación queda integrada al proceso de aprendizaje, me quedo con la firme intención de realizar mis retroalimentaciones más completas de forma que los alumnos puedan aprovechar mejor la materia, al mostrarles claramente el objetivo de la actividad, los aspectos que cumplen de acuerdo a lo solicitado y de qué forma lo realizado les puede servir para actividades posteriores.
\end{abstract}

Parece extraño pero los profesores no siempre están conscientes del porqué se incluyen cada una de las actividades de aprendizaje, pareciera que las revisan como actividades aisladas sin tener un sentido del todo, sin saber hacia dónde se dirigen, sin saber de qué manera cada actividad contribuye a lograr los objetivos del curso, hecho que también quedó evidente entre los comentarios de los participantes, tal como se puede apreciar a continuación:

Las fortalezas tienen que ver con la relación de las actividades desarrolladas por el alumno, con los objetivos de aprendizaje; hacer consciente esto para los alumnos y para mí también, incorporar elementos personales o emoción. Es un modelo que contempla una evaluación integral, me hace consciente que en cada actividad se va construyendo el aprendizaje.

\title{
CONCLUSIONES
}

Ante la pregunta de investigación ¿De qué manera un enfoque de evaluación formativa configura nuevas maneras de retroalimentar en profesores de cursos en línea? Podemos decir que los profesores reconocieron diferencias en su estilo de retroalimentar con referencia al modelo de retroalimentación sugerido por Hattie y Timperley (2007), reconocieron elementos para incluir en su retroalimentación como el ubicar al estudiante en su desempeño con respecto a la meta de aprendizaje, mencionar el objetivo o la competencia de la actividad que se está retroalimentando, decir en qué medida se está contribuyendo a ese objetivo o competencia, informar si el procedimiento que el alumno llevó a cabo para realizar la actividad fue el correcto, y enlazar la actividad que se está retroalimentando con la siguiente actividad.

Los participantes actualizaron retroalimentaciones que habían realizado en períodos anteriores en las que se evidenció las diferencias al proveer información a los estudiantes respondiendo a las preguntas: ¿Hacia dónde voy?, ¿Cómo lo estoy haciendo? y ¿Qué sigue después? 
El sentir de los participantes es que el aprender el modelo de retroalimentación de Hattie y Timperley les motiva a hacer cambios en la forma de retroalimentar, y como expresó uno de los participantes: "nunca imaginé que podría aprender algo nuevo en la forma de retroalimentar, pensé que la retro que les daba a los chavos era de lo mejor, ahora veo que siempre hay cosas por aprender y por mejorar".

Retroalimentar está tomando un nuevo sentido, los profesores hacen consciente que retroalimentar es parte del proceso de enseñanza-aprendizaje, no es solo un recurso para evaluar una actividad de aprendizaje como "bien" o "mal", sino que es información que se le proporciona al estudiante con el fin de apoyarle para que se apropie del aprendizaje que se busca. Retroalimentar en el sentido visto en este estudio es un proceso que siempre será realizado por personas, el tipo de retroalimentación que se propuso en este estudio no puede ser llevado a cabo por ninguna computadora ya que son precisamente las personas las que podemos acompañar al alumno en su proceso de aprendizaje.

Los profesores que participaron en el estudio ya están haciendo cambios en la forma de retroalimentar. Esto ha motivado a los directivos de la institución a seguir ofreciendo este curso de capacitación a sus profesores con el fin de motivarlos, persuadirlos a valorar la importancia que tiene el retroalimentar bajo este nuevo enfoque.

Retroalimentar es proveer información que ayude al estudiante para aprender. Es la idea básica que queremos dejar en el lector de este escrito.

\section{REFERENCIAS BIBLIOGRÁFICAS}

Bonnel, W.; Boehm, H. (2011). Improving Feedback to Students Online: Teaching Tips From Experienced faculty. The Journal of Continuing Education in Nursing, 42 (11), (503-509).

Evans, C. (2013). Making Sense of Assessment Feedback in Higher Education. Review of Educational Research, 83, 1, (70-12).

Gallardo, K. (2012). Evaluación del aprendizaje: retos y mejores prácticas (eBook). Monterrey, México: Editorial Digital del Tecnológico de Monterrey.

Gay, L. R.; Mills, G.; Airasian P. (2006). Educational Research: Competencies for Analysis and Applications. 8th ed.
Upper Saddle River, NJ, EUA: Pearson / Merrill / Prentice Hall.

Hattie, J.; Timperley, H. (2007). The Power of Feed Back. Review of Educational Research, 77 (1), (81-112).

Holmes, K.; Papageorgiou, G. (2009). Good, bad and insufficient: Student's expectations, perceptions and uses of feedback. Journal of Hospitality, Leisure, Sport and Tourisim Education, 8 (1), (85-96).

ITESM. (2013). Portal de la Universidad Tec Virtual. [en línea] Disponible en: http:// www.ruv.itesm.mx/

Jonsson, A. (2012). Facilitating productive use of feedback in higher education. 
Active learning in higher education, 14 (1), 63-76.

Lipnevich, A.; Smith, J. (2009). I really need feedback to learn: students's perspectives on the effectiveness of the differential feedback messages, 21, (347367).
Mathison, S. (1988). Why Triangulate? Educational Researcher. Washington, DC: AERA.

Shute, V. (2008). Focus on formative feedback. Review of Educational Research, 78 (1), (153-189).

Taylor, S. J.; Bogdan, R. (1987). Introducción a los métodos cualitativos de investigación. España: Paidós.

\title{
PERFIL PROFESIONAL Y ACADÉMICO DE LOS AUTORES
}

Fernando Gustavo Lozano Martínez. Licenciado en Ciencias e la Comunicación por el Tecnológico de Monterrey, Maestro en Educación Superior, Universidad Regiomontana. Profesor de la Escuela de Graduados en Educación del Sistema Tecnológico de Monterrey. Instructor de la técnica Aprendizaje Basado en Proyectos (POL), certificado por la Universidad de Twente en Enschede Holanda, certificación en ética por el Tecnológico de Monterrey. Pertenece a la Cátedra de Innovación en Tecnología y Educación (http://www.ruv.itesm.mx/convenio/ catedra/)

E-mail: fernando.lozano@tecvirtual.mx

Laura Adriana Tamez Vargas. Ingeniero Industrial y de Sistemas por el Tecnológico de Monterrey, Maestro en Administración, Tecnológico de Monterrey. Profesor de la Escuela de Graduados en Educación del Sistema Tecnológico de Monterrey. Certificación en la Estrategia Didáctica de Aprendizaje Basado en Problemas para Ingeniería y Ciencias Básicas y Certificación en el Programa de Desarrollo de Habilidades Docentes, basado en competencias, por el Tecnológico de Monterrey.

E-mail: lauratamez@itesm.mx

\section{DIRECCIÓN POSTAL DE LOS AUTORES}

\author{
República Dominicana \#656 \\ Colonia Vistahermosa, \\ CP 66420, Monterrey, N.L. México \\ Balcón del Río \#3020 \\ Colonia Balcones de Altavista, \\ CP 64770, Monterrey, N.L. México
}


Fecha de recepción del artículo: $28 / 10 / 13$

Fecha de aceptación del artículo: 13/02/14

\section{Como citar este artículo:}

Lozano Martínez, F. G.; Tamez Vargas, L. A. (2014). Retroalimentación formativa para estudiantes de educación a distancia. RIED. Revista Iberoamericana de Educación a Distancia, volumen 17, nº 2, pp. 197-221. 